\title{
Consistent Map Building Based on Sensor Fusion for Indoor Service Robot
}

\author{
Ren C. Luo and Chun C. Lai \\ Intelligent Robotics and Automation Lab, National Taiwan University \\ Taipei, Taiwan
}

\section{Introduction}

Consider the actual applications of an intelligent service robot (ISR), it is expected that an ISR will not only autonomously estimate the environment structure but also detect the meaningful symbols or signs in the building it services. For example, an ISR has to locate all the docking stations for recharging itself. For an ISR to lead a customer in the department store to any location such as the toy department or the nearest restroom, it must have the essential recognizing and guiding ability for its service. For this purpose, to carry out an applicable self-localization and map building technique for the indoor service robot becomes important and desirable.

In recent years the sensing and computing technology have made tremendous progress. Various simultaneous localization and mapping (SLAM) techniques have been implemented. The principle of SLAM is derived from Bayesian framework. The EKF-SLAM (DurrantWhyte \& Bailey, 2006) is based on robot state estimation. However, EKF-SLAM will fail in large environments caused by inconsistent estimation problem from the linearization process (Rodriguez-Losada et al., 2006) (Bailey et al., 2006) (Shoudong \& Gamini, 2007). A full SLAM algorithm is using sequential Monte Carlo sampling method to calculate robot state as particle filter (Montemerlo et al., 2002) (Montemerlo et al., 2003). But the technique will grow exponentially with the increase of dimensions of the state space. Another full scan matching method is suitable for the environment reconstruction ( $\mathrm{Lu} \&$ Milios, 1997) (Borrmann et al., 2008). But the pose variable will also grow enormously depending on the sampling resolution.

Based on the practical needs of a service robot patrol in the building, it is desirable to construct an information map autonomously in a unitary SLAM process. This chapter investigates a consistent map building by laser rangefinder. Firstly, the Covariance Intersection (CI) method is utilized to fuse the robot pose for a robust estimation from wheel encoder and laser scan match. Secondly, a global look up table is built for consistent feature association and a global fitness function is also generated. Finally, the Particle Swarm Optimization (PSO) method is applying to solve the optimal alignment problem. From the proposed method, a consistent map in a unitary localization and mapping process via the sensor fusion and optimal alignment methodology has been constructed and implemented 
successfully. Furthermore, a complete 2.5D environment map will be constructed rapidly with the Mesa SwissRanger (Sr, 2006).

\section{Robot Pose Estimation}

\subsection{Covariance Intersection on Sensor Fusion}

The Covariance Intersection (CI) is a data fusion algorithm which takes a convex combination of the means and covariance in the information space. The major advantage of $\mathrm{CI}$ is that it permits filter and data fusion to be performed on probabilistically defined estimates without knowing the degree of correlation among those estimates. Consider two different pieces of measurement $A$ and $B$ from different sources. If given the mean and variance: $\mathrm{E}\{\mathrm{A}\}=\mathrm{a}, \mathrm{E}\{\mathrm{B}\}=\mathrm{b}, \operatorname{var}\{\mathrm{A}, \mathrm{A}\}=\mathrm{P}_{\mathrm{aa}}, \operatorname{var}\{\mathrm{B}, \mathrm{B}\}=\mathrm{P}_{\mathrm{bb}}, \operatorname{cov}\{\mathrm{A}, \mathrm{B}\}=\mathrm{P}_{\mathrm{ab}}$ Define the estimate as a linear combination of $\mathrm{A}$ and $\mathrm{B}$ where are present the previous estimate of the same target with certain measurement uncertainty. The CI approach is based on a geometric interpretation of the Kalman filter process. The general form of the Kalman filter can be written as:

$$
\begin{gathered}
\hat{\mathrm{z}}=\omega_{\mathrm{a}} \mathrm{a}+\omega_{\mathrm{b}} \mathrm{b} \\
\mathrm{P}_{\mathrm{zz}}=\omega_{\mathrm{a}} \mathrm{P}_{\mathrm{aa}} \omega_{\mathrm{a}}{ }^{\mathrm{T}}+\omega_{\mathrm{a}} \mathrm{P}_{\mathrm{ab}} \omega_{\mathrm{a}}{ }^{\mathrm{T}}+\omega_{\mathrm{b}} \mathrm{P}_{\mathrm{ba}} \omega_{\mathrm{b}}{ }^{\mathrm{T}}+\omega_{\mathrm{b}} \mathrm{P}_{\mathrm{bb}} \omega_{\mathrm{b}}{ }^{\mathrm{T}}
\end{gathered}
$$

where the weights $\omega_{\mathrm{a}}$ and $\omega_{\mathrm{b}}$ are chosen to minimize $\mathrm{P}_{\mathrm{zz}}$.

This form reduces to the conventional Kalman filter if the estimates are independent $\left(P_{a b}=0\right)$. The covariance ellipsoid of $C I$ will enclose the intersection region and the estimate is consistent. CI does not need assumptions on the dependency of the two pieces of information when it fuses them. Given the upper bounds $\mathrm{P}_{\text {aa }}-\overline{\mathrm{P}}_{\mathrm{aa}} \geq 0$ and $\mathrm{P}_{\mathrm{bb}}-\overline{\mathrm{P}}_{\mathrm{bb}} \geq 0$, the covariance intersection estimate output are defined as follows:

$$
\begin{gathered}
\mathrm{z}=\mathrm{P}_{\mathrm{zz}}\left\{\omega_{\mathrm{a}} \mathrm{P}_{\mathrm{aa}}^{-1} \mathrm{a}+\omega_{\mathrm{b}} \mathrm{P}_{\mathrm{bb}}{ }^{-1} \mathrm{~b}\right\} \\
\mathrm{P}_{\mathrm{zz}}{ }^{-1}=\omega_{\mathrm{a}} \mathrm{P}_{\mathrm{aa}}^{-1}+\omega_{\mathrm{b}} \mathrm{P}_{\mathrm{bb}}^{-1}
\end{gathered}
$$

where $\omega_{\mathrm{a}}+\omega_{\mathrm{b}}=1 \quad, \quad 0 \leq \omega_{\mathrm{a}}, \omega_{\mathrm{b}} \leq 1$

The parameter $\omega$ modifies the relative weights assigned to A and B. Different choices of $\omega$ can be used to optimize the covariance estimate with respect to different performance criteria such as minimizing the trace or the determinant of $\mathrm{P}_{\mathrm{zz}}$. Let $\alpha \equiv \sqrt{\operatorname{tr}\left\{\omega_{\mathrm{a}} \mathrm{P}_{\mathrm{aa}} \omega_{\mathrm{a}}{ }^{\mathrm{T}}\right\}}$ $\beta \equiv \sqrt{\operatorname{tr}\left\{\omega_{\mathrm{b}} \mathrm{P}_{\mathrm{bb}} \omega_{\mathrm{b}}{ }^{\mathrm{T}}\right\}}$

$$
\mathrm{P}_{\mathrm{zz}}=\frac{\alpha}{\alpha+\beta} \mathrm{P}_{\mathrm{aa}}^{-1}+\frac{\beta}{\alpha+\beta} \mathrm{P}_{\mathrm{bb}}^{-1}
$$




$$
\omega_{\mathrm{a}}=\frac{\alpha}{\alpha+\beta} \mathrm{P}_{\mathrm{zz}} \mathrm{P}_{\mathrm{aa}}^{-1} \quad \omega_{\mathrm{b}}=\frac{\beta}{\alpha+\beta} \mathrm{P}_{\mathrm{zz}} \mathrm{P}_{\mathrm{bb}}^{-1}
$$

This theorem reveals the nature of the optimality of the best $\omega$ in $\mathrm{CI}$ algorithm. The CI algorithm also provides a convenient parameterization for the optimal solution in n-square dimensional space. The results can be extended to multiple variables and partial estimates as below:

$$
\begin{aligned}
& \mathrm{z}=\mathrm{P}_{\mathrm{zz}}\left(\omega_{1} \mathrm{~A}_{1}^{-1} \mathrm{a}_{1}+\omega_{2} \mathrm{~A}_{2}^{-1} \mathrm{a}_{2}+\ldots+\omega_{\mathrm{n}} \mathrm{A}_{\mathrm{n}}{ }^{-1} \mathrm{a}_{\mathrm{n}}\right) \\
& \mathrm{P}_{\mathrm{zz}}{ }^{-1}=\omega_{1} \mathrm{~A}_{1}^{-1}+\omega_{2} \mathrm{~A}_{2}^{-1}+\omega_{3} \mathrm{~A}_{3}^{-1}+\ldots+\omega_{\mathrm{n}} \mathrm{A}_{\mathrm{n}}{ }^{-1}
\end{aligned}
$$

where $\left\{\mathrm{a}_{\mathrm{i}}, \mathrm{A}_{\mathrm{i}}\right\}$ refers to the $\mathrm{i}$-th measurement input and $\sum_{\mathrm{i}=1}^{\mathrm{n}} \omega_{\mathrm{i}}=1$

\subsection{Sequence Robot Pose Uncertainty Representation}

When a robot platform is moving, the encoder will provide precision pulse resolution for motor control. Unfortunately, the medium between servomotor and floor is not rigid so that errors will occur on robot pose estimation. A Gaussian prior probability may be tried to represent the pose uncertainty from encoder transformation. For a sequence robot pose uncertainty representation, Fig. 1 (a) shows that robot is moving along the dash line.
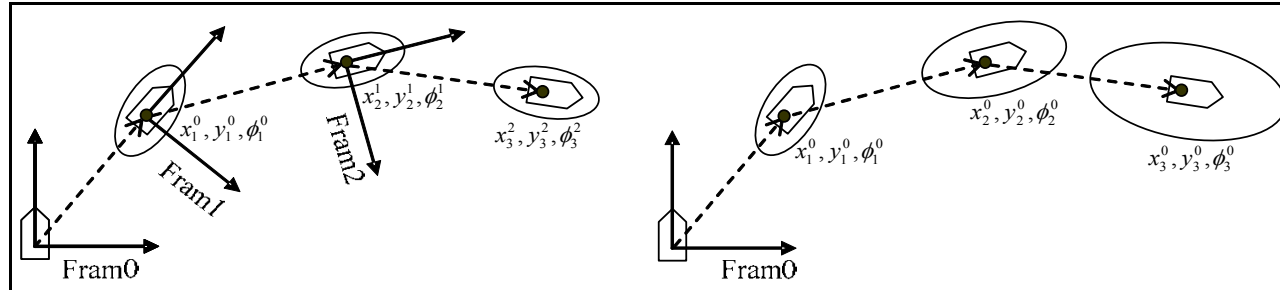

(a)

(b)

Fig. 1. Robot Sequence Pose Estimation and Uncertainty Representation (a) Robot pose uncertainty in opposition to last frame (b) Robot pose uncertainty in opposition to original frame

Each pose uncertainty variation is respect to last local frame or time index. For convenience, the ellipse only represents the Gaussian covariance uncertainty in two-dimension position estimation. The pose $\left(x_{3}^{2}, y_{3}^{2}, \phi_{3}^{2}\right)$ is the mean in the third measurement respect to frame 2 and so on. The problem is that measurement sequence will produce accumulated error respect to original Frame 0 as shown in Fig. 1 (b). A compound mean and error covariance transformation can be derived from previous estimation in expansion matrix form as: 


$$
\begin{gathered}
{\left[\begin{array}{l}
x_{3}^{0} \\
y_{3}^{0} \\
\phi_{3}^{0}
\end{array}\right]=\left[\begin{array}{c}
x_{2}^{1} \cos \phi_{1}^{0}-y_{2}^{1} \sin \phi_{1}^{0}+x_{1}^{0} \\
x_{2}^{1} \sin \theta_{1}^{0}-y_{2}^{1} \cos { }_{1}^{0}+y_{1}^{0} \\
\phi_{1}^{0}+\phi_{2}^{1}
\end{array}\right]} \\
C_{0}^{3}=J\left(\begin{array}{cc}
C_{1}^{0} & 0 \\
0 & C_{2}^{1}
\end{array}\right) J^{T}
\end{gathered}
$$

where $J$ is the Jacobian of the transformation at the mean values variables:

$$
J=\left(\begin{array}{cccccc}
1 & 0 & -\left(y_{3}^{2}-y_{1}^{0}\right) & \cos \phi_{1}^{0} & -\sin \phi_{1}^{0} & 0 \\
0 & 1 & \left(x_{3}^{2}-x_{1}^{0}\right) & \sin \phi_{1}^{0} & \cos \phi_{1}^{0} & 0 \\
0 & 0 & 1 & 0 & 0 & 1
\end{array}\right)
$$

\subsection{Pose Estimation from ICP Alignment}

In $3 \mathrm{D}$ shapes registration application, the iterative closest point (ICP) algorithm was successful apply to align two given point data sets. The ICP algorithm was developed by (Besl \& McKay, 1992) and the principle works as follows. Let $P_{0}=\left\{p_{1}, \ldots, p_{m}\right\}$ represent the observation point set and $P_{r}=\left\{p_{1}, \ldots, p_{n}\right\}$ be the reference point set. The object of the algorithm is to find a geometric transformation to align the observed point $P_{0}$ to the reference point set $P_{r}$. This transformation is composed of rotation and translation matrix $(\mathrm{R}, \mathrm{T})$. (Nieto et al., 2007) took the algorithm as an association criterion of EKF-SLAM because ICP algorithm makes the association strengthened using the shape as a gate criterion. In this section, the ICP result is regarded as a sensor output on pose estimation between two adjacent measurements from laser ranger. The error covariance evolution on the ICP alignment can be derived as follows:

$$
\begin{gathered}
z=\left\{\rho_{i}, \theta_{i}\right\}, \rho_{i}=r_{i}+\varepsilon \\
\left.P_{i}=\left[\rho_{i} \cos \theta_{i}, \rho_{i} \cos \theta_{i}\right]^{T}\right\}, i=1 \ldots n \\
I=\sum_{i}\left\|\left(R \cdot p_{i}^{k}+T\right)-\operatorname{map}\left(R \cdot p_{i}^{k}+T, P^{k-1}\right)\right\|
\end{gathered}
$$

where $k$ represents the frame or time index and function map( ) maps the data points $p_{i}$ in frame $k$ into the model points in frame $k-1$. The ICP algorithm always can find out the transform if the error function can be minimized within a threshold, i.e., ICP arrives in a fit solution. Under this constraint, the covariance approximation depends only on the error function $I$ being minimized and the term $\partial^{2} I / \partial Z \partial X$ addresses variation of the error function caused by measurement noise. Therefore, the covariance of pose transformation is represented as: 


$$
\operatorname{cov}(X) \cong\left(\frac{\partial^{2} I}{\partial x^{2}}\right)^{-1} \frac{\partial^{2} I}{\partial Z \partial X} \operatorname{cov}(Z) \frac{\partial^{2} I}{\partial Z \partial X}\left(\frac{\partial^{2} I}{\partial X^{2}}\right)^{-1}
$$

where $Z$ is from laser measurement and $X$ is the pose transformation. In equation (14), the Cramér-Rao lower bound constraint is proven satisfied (Censi, 2007).

\subsection{Multi-Pose Estimation Using $\mathrm{Cl}$}

For real sensor data acquisition in this study, the sampling rate of encoder is higher than the laser ranger due to higher post-acquisition process requirements of laser ranger. Thus, time sequence alignment is required before fusion process. The encoder uncertainty can be derived by an independent experiment as an approximate covariance matrix. With time sequence index, the uncertainty compound process is needed. When the latest pose estimate is obtained from laser ranger in current time frame, the covariance intersection method will be applied. Fig. 2 shows the concept and the solid ellipse shows the optimal fusion result. In Fig. 3 , the actual CI process resulting from robot pose translation is represented. The mobile robot was manipulated to go forward in 50 centimeter (positive y axis on robot local frame) or rotate at each sampling time. The blue line in Fig. 3 shows a pre-determined uncertainty with a 2-deviation on robot $x-y$ translation in each time index. Taking CI fusion with the result from the pre-determined uncertainty of encoder and ICP result from equation (14), the new $\mathrm{CI}$ mean is the magenta circle and the magenta line represents the new $\mathrm{CI}$ deviation. From the new CI result, a less uncertain interval (the black line) is obtained, i.e., the new estimation will be more close to the true translation (the black star) as shown in Fig. 3.

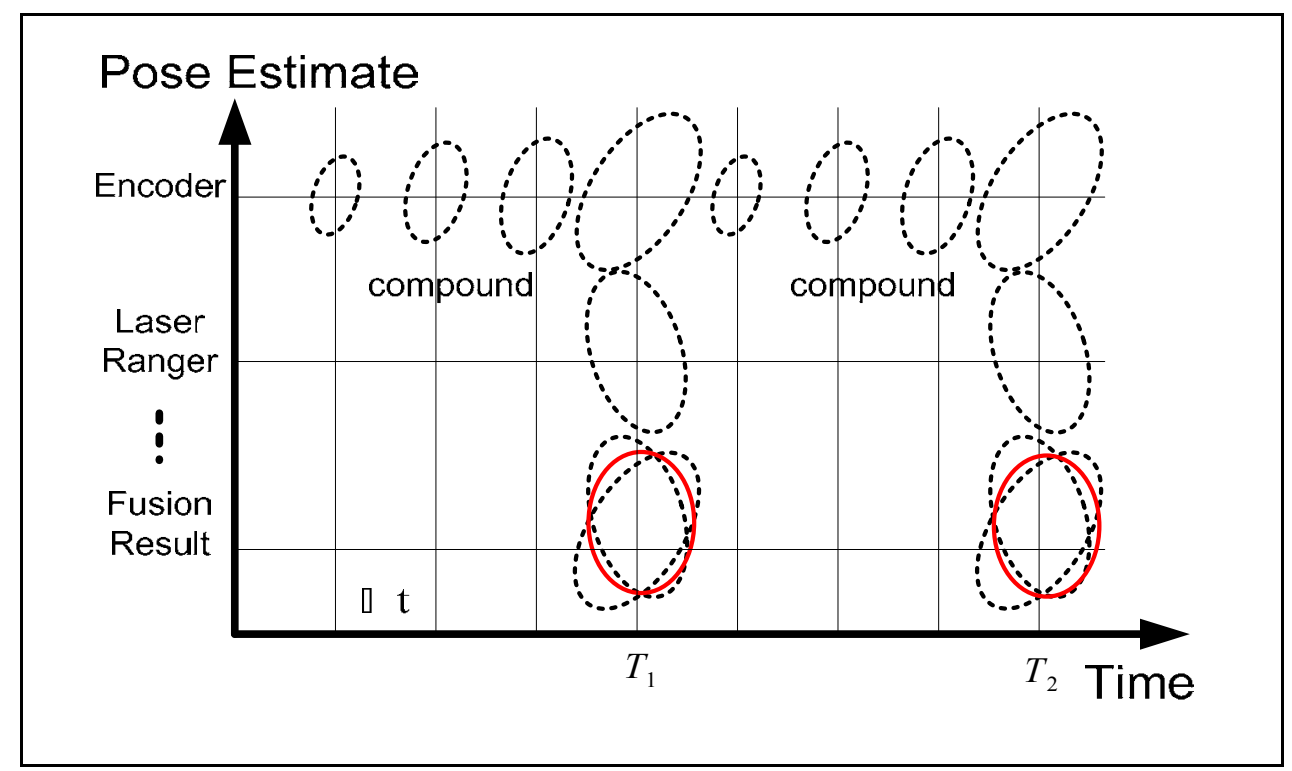

Fig. 2. Position Estimation Using Covariance Intersection 


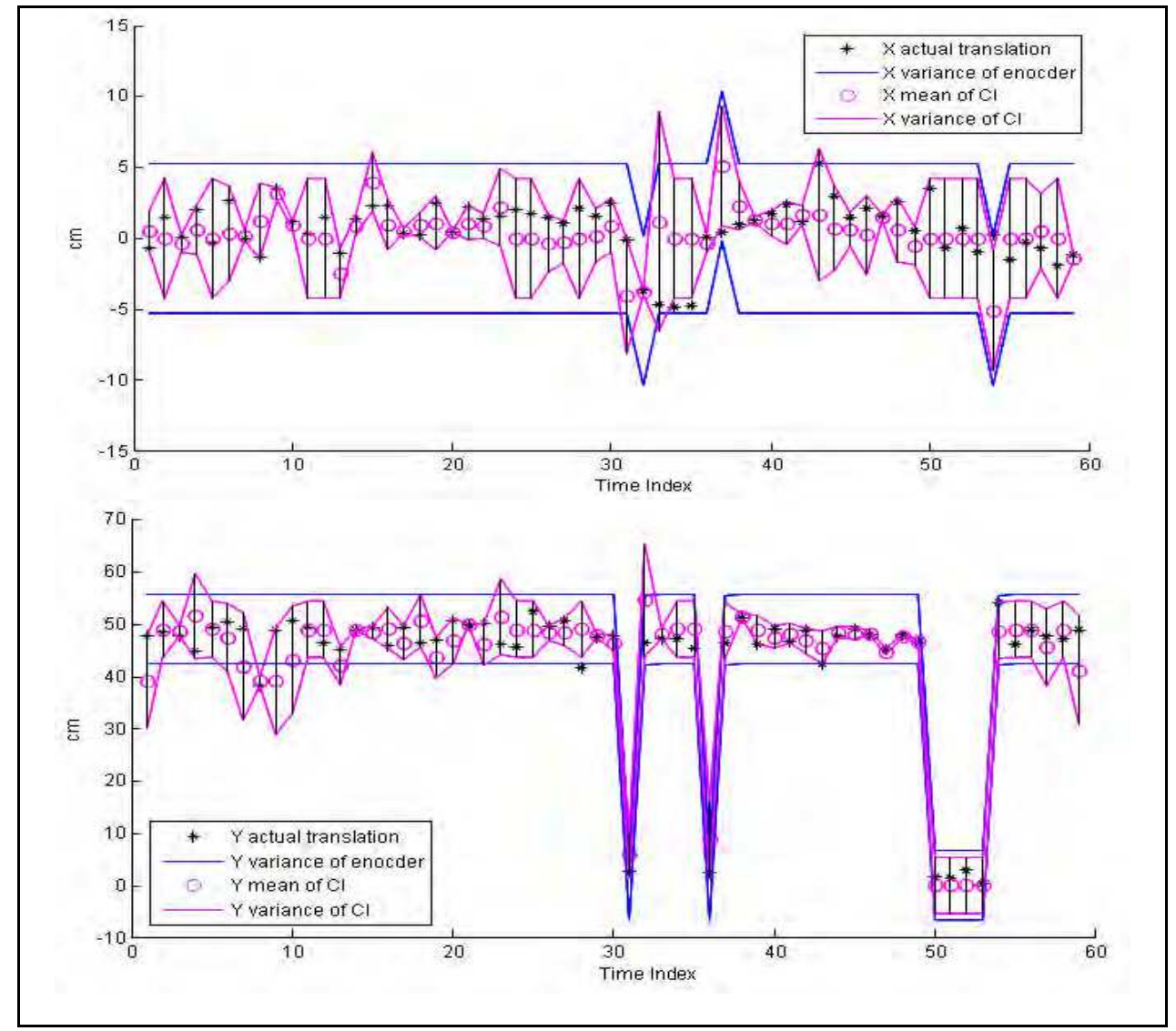

Fig. 3. Covariance Intersection Fusion Result on Robot Pose Translation

\section{Consistent Map Alignment}

\subsection{Segment Extraction from Laser Measurement}

For building a consistent geometry map, the distinctive landmarks should be identified and extracted first. Since most of the indoor environment can be efficiently described using polygon segments. As the geometry features are defined based on line segments. From each laser ranger measurement $s=\left\{p_{0}, p_{1}, \ldots, p_{n-1}, p_{n}\right\}$, the Iterative End Point Fit (IEPF) (Borges, \& Aldon, 2004) method is applied ahead. The IEPF recursively splits $S$ into two subsets $s_{1}=\left\{p_{0}, \ldots, p_{j}\right\}$ and $s_{2}=\left\{p_{j}, \ldots, p_{n}\right\}$ while a validation criterion distance $d_{\max }$ is satisfied from point $p_{j}$ to the segment between $p_{0}$ and $p_{n}$. Through the iteration, IEPF function will return all segment endpoints $\left\{p_{0}, p_{j}\right\} 、\left\{p_{j}, p_{n}\right\}$. However, IEPF only renders cluster points for each segment as candidate. For more precision line segment estimation, a Linear Regression (LR) method is used to estimate the line equation from each segment candidate. 
Fig. 4 (a) shows the laser measurement. In Fig. 4 (b), the starred points are IEPF results and Fig. 4 (c) shows the segment extraction after LR extraction.

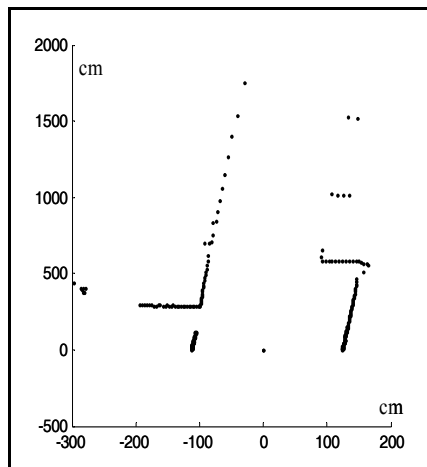

(a)

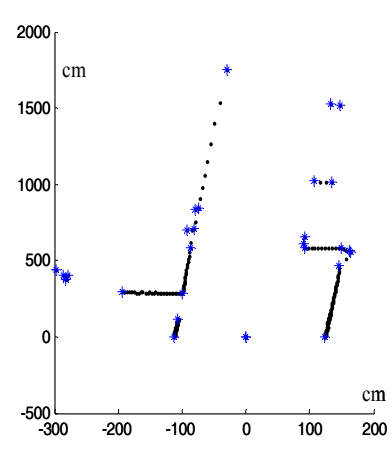

(b)

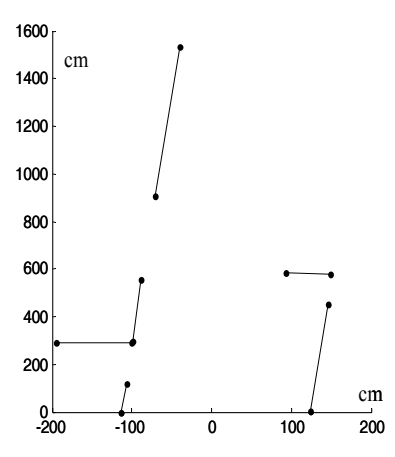

(c)

Fig. 4. (a) Laser ranger measurement (b) IEPF result from laser measurement (c) Segment extraction result

\subsection{Consistent Association and mapping}

The objective of the data association is to eliminate the accumulated error from measurements. The issue is focused on having an accuracy link of landmarks between current and previous observations. From the physical continuity of robot motion, the adjacent measurement of the environment will have the maximum correlation. Also, the ICP method will reach the maximum matching criterion based on the adjacent measurement. Combining encoder measurements in above section, the robust pose estimation is achieved between the adjacent laser measurements. Fig. 5 (a) shows two adjacent laser scans based on robot center. Fig. 5 (b) shows two adjacent laser scans after the pose fusion result.

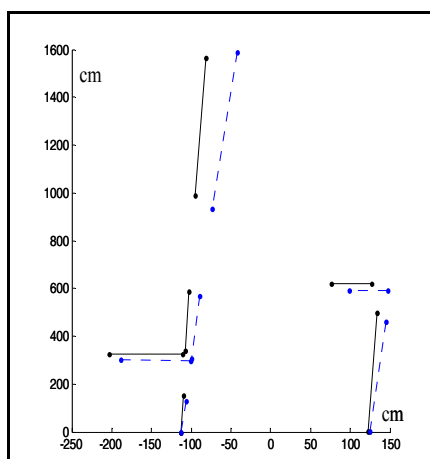

(a)

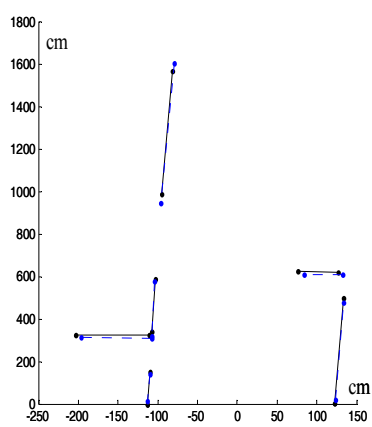

(b)

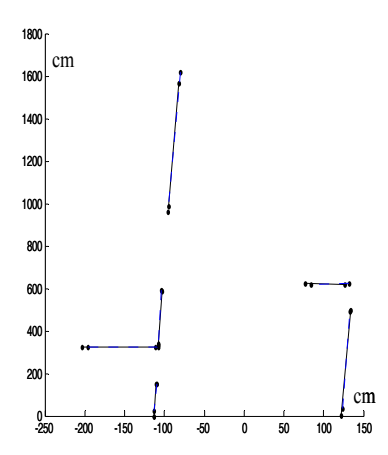

(c)

Fig. 5. (a) Segment extraction on two adjacent pose, the solid is model and the dash is new data (b) Fusion result on adjacent pose variation (c) Pose alignment after PSO 
If there are $r$ solid segments in previous frame $n-1$ and there are s dash segments in current frame $n$. A data association criterion is built based on the adjacent segment distance as below:

$$
\begin{aligned}
& \text { if } \operatorname{dist}\left(\operatorname{seg}_{i \in r}^{n-1}, \operatorname{seg}_{j \in s}^{n}\right)<\text { threshold } \\
& \qquad \operatorname{seg}_{j \in s}^{n} \text { is mapping to } \operatorname{seg}_{i \in r}^{n-1} \\
& \text { esle } \\
& \quad \operatorname{seg}_{j \in s}^{n} \text { is a new landmark }
\end{aligned}
$$

Via the criterion, the global data association will be connected by successive mapping. Furthermore, the global feature will grow up when a new segment feature is observed. Table I represents the "Association Look Up Table". In measurement frame 0, three segments 1, 2 and 3 are identified and transferred to global features as 1-th, 2-th and 3-th. In frame 1, three segments 1,2 , and 3 are extracted and the first two segments 1 and 2 are mapped to segments 2 and 3 in previous frame 0 by the association criterion. So the segments 1 and 2 in frame 1 will associate to the 2-th and 3-th in global and the segments 3

\begin{tabular}{|c|c|c|c|c|c|c|c|c|c|c|c|}
\hline $\begin{array}{l}\text { MEASUREMENT } \\
\text { FRAME } \\
\end{array}$ & \multicolumn{5}{|c|}{$\begin{array}{l}\text { ADJACENT } \\
\text { MAPPING }\end{array}$} & \multicolumn{6}{|c|}{$\begin{array}{c}\text { GLOBAL } \\
\text { ASSOCIATION }\end{array}$} \\
\hline 0 & 1 & 2 & 3 & & & $\underline{1}$ & $\underline{2}$ & $\underline{3}$ & & & \\
\hline 1 & $\begin{array}{l}\nearrow \\
1\end{array}$ & $\begin{array}{l}\nearrow \\
2\end{array}$ & 3 & & & & 2 & 3 & $\underline{4}$ & & \\
\hline 2 & $\begin{array}{l}\uparrow \\
1\end{array}$ & $\begin{array}{l}\uparrow \\
2\end{array}$ & $\begin{array}{l}\uparrow \\
3\end{array}$ & & & & 2 & 3 & 4 & & \\
\hline 3 & $\begin{array}{l}\uparrow \\
1\end{array}$ & $\begin{array}{l}\uparrow \\
2\end{array}$ & $\begin{array}{l}\uparrow \\
3\end{array}$ & 4 & & & 2 & 3 & 4 & $\underline{5}$ & \\
\hline$\ldots \ldots$ & $\begin{array}{l}\uparrow \\
1\end{array}$ & $\begin{array}{l}\uparrow \\
2\end{array}$ & $\begin{array}{l}\uparrow \\
3\end{array}$ & $\begin{array}{l}\uparrow \\
4\end{array}$ & 5 & & & 3 & 4 & 5 & $\underline{6}$ \\
\hline$n-1$ & $\begin{array}{l}T \\
1\end{array}$ & $\begin{array}{l}\nearrow \\
2\end{array}$ & $\begin{array}{l}7 \\
3\end{array}$ & $\begin{array}{l}7 \\
4\end{array}$ & & & & 3 & 4 & 5 & 6 \\
\hline$n$ & $\begin{array}{l}\uparrow \\
1\end{array}$ & $\begin{array}{l}\uparrow \\
2\end{array}$ & $\begin{array}{l}\uparrow \\
3\end{array}$ & $\begin{array}{l}\uparrow \\
4\end{array}$ & & & & 3 & 4 & 5 & 6 \\
\hline
\end{tabular}
in frame 1 will create a new landmark as the 4-th in global features. In frame n, there are four segments map to frame n-1 and these four segments are associated in global from the 3th to the 6-th.

Table 1. Association Look Up Table

In order to eliminate the residual error accumulated from pose estimation, a global fitness function is generating based on the global association via the association look up table. The fitness function is composed of Euclidean distance between the all segments that associated to the primitive global segments. 


$$
\text { fitness }=\sum_{i=1}^{k} \frac{\left|a_{i} x_{i}^{1}+b_{i} y_{i}^{1}+c_{i}\right|+\left|a_{i} x_{i}^{2}+b_{i} y_{i}^{2}+c_{i}\right|}{\sqrt{a_{i}+b_{i}}}
$$

where $k$ is the quantity of the segment mapping between the adjacent frames. The $a_{i}, b_{i}$ and $c_{i}$ are the corresponding segment parameters in global frame and $\left(x_{i}, y_{i}\right)$ are the endpoints which are translated by current robot pose.

\subsection{Pose Alignment Using Particle Swam Optimization}

The PSO technique was proposed by (Eberhart \& Kennedy, 1995) has been widely used in finding solutions for multi-variable optimization problems. Some improvements and applications have also been proposed (Shi \& Eberhart, 1998) (Stacey et al., 2003) (Zwe-Lee, 2003). It maintains several particles (each represents a solution) and simulates the behavior of bird flocking to find the final solutions. All the particles continuously move in the search space, toward better solutions, until the termination criteria are met. After certain iterations, the optimal solution or an approximate optimal solution is expected to be found. When applying the PSO technique, each possible solution in the search space is called a particle, which is similar to a bird's move mentioned above. All the particles are evaluated by a fitness function, with the values representing the goodness degrees of the solutions. The solution with the best fitness value for a particle can be regarded as the local optimal solution found so far and is stored as $p B e s t$ solution for the particle. The best one among all the pBest solutions is regarded as the global optimal solution found so far for the whole set of particles, and is called the gBest solution. In addition, each particle moves with a velocity, which will dynamically change according to pBest and gBest. After finding the two best values, a particle updates its velocity by the following equation:

$$
\begin{array}{r}
V_{\text {id }}^{\text {new }}=\omega \times V_{\text {id }}^{\text {old }}+ \\
c_{1} \times \text { Rand }_{1}() \times\left(\text { pBest }_{i d}-x_{i d}\right)+ \\
c_{2} \times \text { Rand }_{2}() \times\left(\text { BBest }_{i d}-x_{i d}\right)
\end{array}
$$

where

(a) $V_{i d}^{\text {new }}$ :the velocity of the i-th particle in the d-th dimension in the next generation;

(b) $V_{\text {id }}^{\text {old }}$ :the velocity of the i-th particle in the d-th dimension in the current generation;

(c) B Best $_{i d}$ : the current pBest value of the i-th particle in the d-th dimension;

(d) gBest $_{i d}$ : the current gBest value of the whole set of particles in the d-th dimension;

(e) $x_{i d}$ the current position of the i-th particle in the d-th dimension;

(f) $\omega$ : the inertial weight;

(g) $c_{1}$ : the acceleration constant for a particle to move to its $p B e s t$;

(h) $c_{2}$ : the acceleration constant for a particle to move to the $g B e s t_{i d}$;

(i) $\operatorname{Rand}_{1}(), \operatorname{Rand}_{2}()$ : two random numbers between 0 to 1 .

After the new velocity is found, the new position for a particle can then be obtained as: 


$$
x_{i d}^{\text {new }}=x_{i d}^{\text {old }}+V_{i d}^{\text {new }}
$$

The proposed approach works well to find out the optimal fitness based on segments alignment. The pose fusion result from encoder and ICP method described in section 2 gives a good initial guess on the optimal search as shown in Fig. 5 (b). Fig. 5 (c) shows the optimal alignment result after PSO. Fig. 6 shows performance comparison of Mathwork Optimal Toolbox (Coleman et al., 1999) and PSO algorithm. Both algorithms were given the same initial guess value with a normalized iteration time. Three fitness functions on two, four and seven alignment conditions are evaluated to find out the global minimum and five particles are predefined in PSO search. The top figure in Fig. 6 shows both optimal algorithms will find out the same global minimum under a 2-alignment fitness constraint. But with more complex fitness condition, the PSO always converge faster and search better than Mathwork function, because the numerical Newton-Raphson technique or direct search (Lagarias et al., 1998) may fall into a local minimum. On the contrary, PSO algorithm has the advantage for global optimal search.

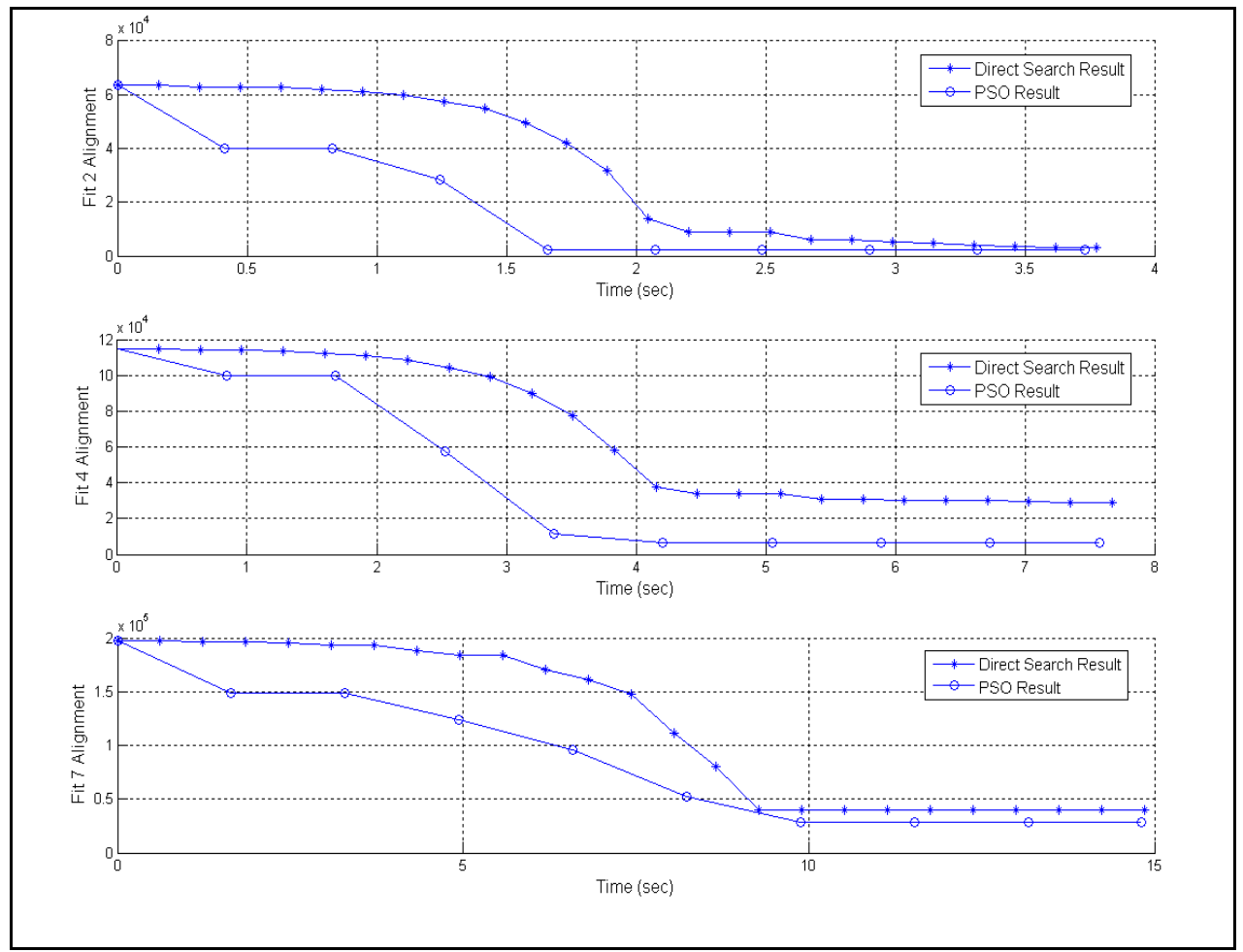

Fig. 6. Performance comparison between Matlab Optimal Toolbox and PSO.

The top shows two segments optimal alignment result, the middle shows four segments optimal alignment result and the bottom shows seven segments optimal alignment result. 


\section{Consistent Map Building Construction}

\subsection{Map Building in a Unitary SLAM Tour}

A SICK LMS-100 laser ranger is quipped in the robot platform as shown in Fig. 7. In each sampling time, the encoder, laser measurement are compounded and recorded. Applying the consistent alignment methodology with the association look up table described in section 3, the robot pose can be optimally corrected in global frame after each measurement. Fig. 8 shows the complete environment map of the corridor with global segment landmarks and the blue rectangles present the global segment landmarks which are created in the look up table.

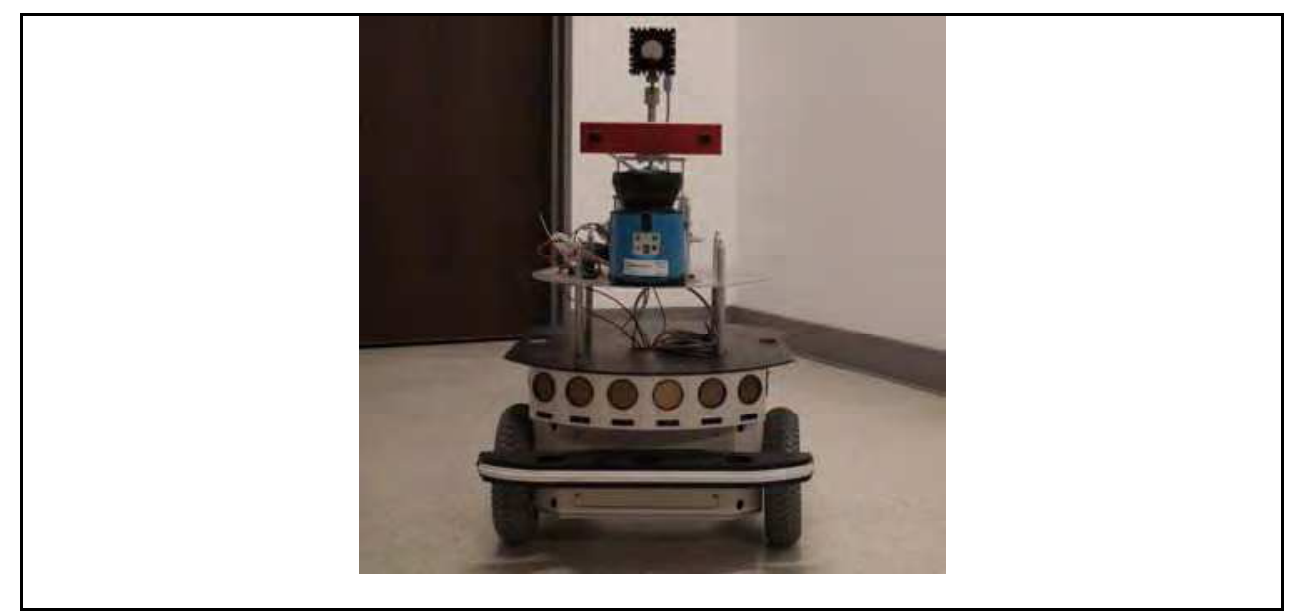

Fig. 7. SICK LMS-100 is equipped on the mobile robot platform

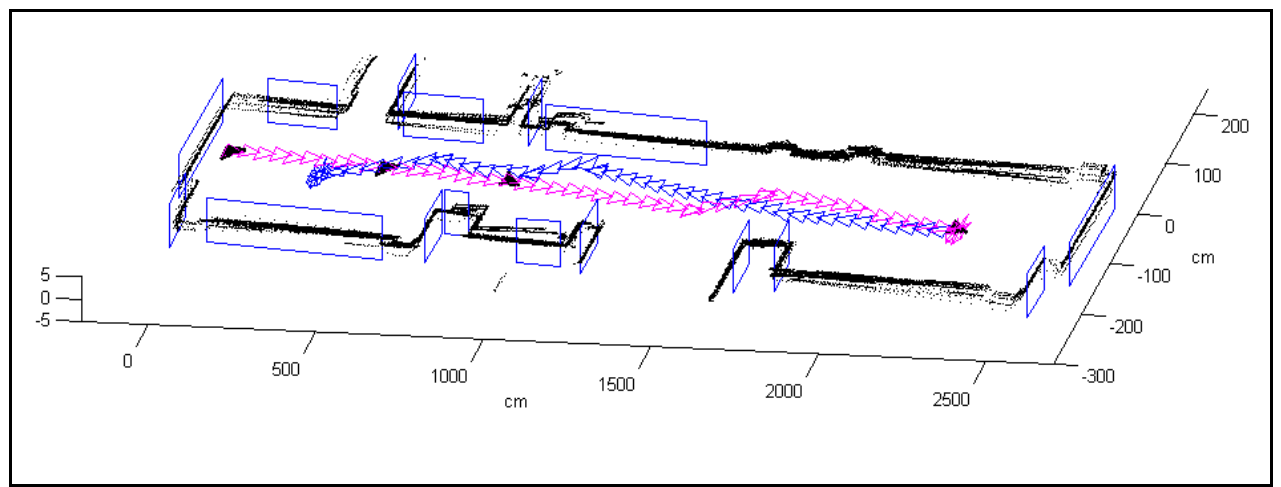

Fig. 8. The consistent map of a corridor with segment landmarks

\subsection{Rapid 2.5D Info-Map Building with Mesa SwissRanger}

In this experiment, a relief environment map is to be built within a SALM tour and the SwissRagner $(\mathrm{Sr}, 2006)$ is applied. SwissRanger belongs to the active 3D sensors. Its measurement is based on a phase-measuring time-of-flight (TOF) principle. A light source 
emits a near-infrared wave front that is intensity-modulated with a few tens of MHz. The light is reflected by the scene and imaged by an optical lens onto the dedicated CCD/CMOS sensor with a resolution in $176 \times 144$ pixels. Depending on the distance of the target, the captured image is delayed in phase compared to the originally emitted light wave. By measuring the phase delay of each image pixel, it will provide amplitude data, intensity data and distance data, which are weakly correlated to each other. All measurements are being organized by a FPGA hardware implement, which provides an USB interface to access the data values. In practical applications, two parameters will influence the measurement results: one is the integration time and the other is the amplitude threshold. If the integration time is short then the results are very noisy and if it is long then the results are getting blurred with moving objects. A suitable calibration on amplitude threshold is also needed, because the amplitude threshold will filter out the noise due to the reflection of the environment components.

The SwissRanger used in the experiment is equipped with a pre-calibrated SICK laser ranger. Following the previous process, the robot pose will be estimated with the consistent map alignment in each time index. With the pre-calibrated SICK laser ranger, the 3D measurements from SwissRanger are available. To execute a calibrated transformation, all the 3D measurements will also keep on the optimal alignment. Fig. 9 shows the complete 2.5D info-map building result with the SwissRanger scanning.

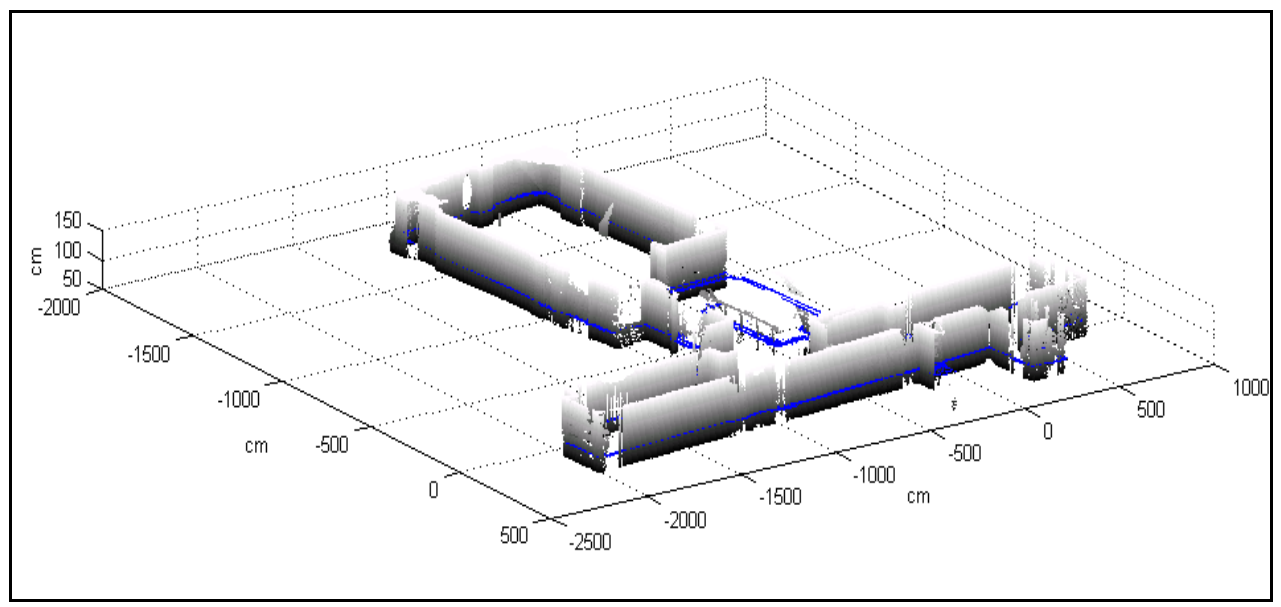

Fig. 9. A complete 2.5D map building with SwissRanger scanning

\section{Conclusion}

This chapter presents a consistent map construction in a unitary SLAM (simultaneously localization and mapping) process through the sensor fusion approach and optimal alignment technologies. The system will autonomously provide the environment geometrical structure for intelligent robot service in a building. In order to build the consistent map, a CI (Covariance Intersection) rule fuses the uncertainty from wheel encoder and ICP (Iterative Closest Point) result as a robust initial value of the fitness function. The alignment fitness function is composed of the Euclidean distances which are associated from 
current features to the global ones by a quick look up table. After applying the artificial PSO (Particle Swarm Optimization) algorithm, the optimal robot pose for the map alignment is obtained. Finally, by employing SwissRanger sensor, a complete $2.5 \mathrm{D}$ info-map cab be rapidly built up for indoor service robot applications.

\section{References}

Bailey, T., Nieto, J., Guivant, J., Stevens, M., \& Nebot, E. (2006). Consistency of the EKFSLAM Algorithm, Intelligent Robots and Systems, 2006 IEEE/RSJ International Conference on, pp. 3562-3568.

Besl, P. J., \& McKay, H. D. (1992). A method for registration of 3-D shapes. Pattern Analysis and Machine Intelligence, IEEE Transactions on, 14, 239-256.

Borges, G. A., \& Aldon, M. J. (2004). Line extraction in 2D range images for mobile robotics. Journal of Intelligent and Robotic Systems, 40, 267-297.

Borrmann, D., Elseberg, J., Lingemann, K., Nuchter, A., \& Hertzberg, J. (2008). Globally consistent 3D mapping with scan matching. Robotics and Autonomous Systems, 56, $130-142$.

Censi, A. (2007). On achievable accuracy for range-finder localization, Robotics and Automation, 2007 IEEE International Conference on, pp. 4170-4175.

Coleman, T., Branch, M. A., \& Grace, A. (1999). Optimization toolbox. For Use with MATLAB. User's Guide for MATLAB 5, Version 2, Relaese II.

Duda, R. O., \& Hart, P. E. (1973). Pattern classification and scene analysis, New York.

Durrant-Whyte, H., \& Bailey, T. (2006). Simultaneous localization and mapping: part I. Robotics \& Automation Magazine, IEEE, 13, 99-110.

Eberhart, R., \& Kennedy, J. (1995). A new optimizer using particle swarm theory, Micro Machine and Human Science, 1995. MHS '95., Proceedings of the Sixth International Symposium on, pp. 39-43.

Kennedy, J., \& Eberhart, R. (1995). Particle swarm optimization, Neural Networks, 1995. Proceedings., IEEE International Conference on, pp. 1942-1948.

Lagarias, J. C., Reeds, J. A., Wright, M. H., \& Wright, P. E. (1998). Convergence properties of the Nelder-Mead simplex algorithm in low dimensions. SIAM Journal on Optimization, 9, 112-147.

Lu, F., \& Milios, E. (1997). Robot pose estimation in unknown environments by matching 2d range scans. Journal of Intelligent and Robotic Systems, 18, 249-275.

Montemerlo, M., Thrun, S., Koller, D., \& Wegbreit, B. (2002). FastSLAM: A factored solution to the simultaneous localization and mapping problem, pp. 593-598, Menlo Park, CA; Cambridge, MA; London; AAAI Press; MIT Press; 1999.

Montemerlo, M., Thrun, S., Koller, D., \& Wegbreit, B. (2003). FastSLAM 2.0: An improved particle filtering algorithm for simultaneous localization and mapping that provably converges, pp. 1151-1156), LAWRENCE ERLBAUM ASSOCIATES LTD.

Nieto, J., Bailey, T., \& Nebot, E. (2007). Recursive scan-matching SLAM. Robotics and Autonomous Systems, 55, 39-49.

Rodriguez-Losada, D., Matia, F., \& Galan, R. (2006). Building geometric feature based maps for indoor service robots. Robotics and Autonomous Systems, 54, 546-558. 
Shi, Y., \& Eberhart, R. (1998). A modified particle swarm optimizer, Evolutionary Computation Proceedings, 1998. IEEE World Congress on Computational Intelligence., The 1998 IEEE International Conference on, pp. 69-73.

Shoudong, H., \& Gamini, D. (2007). Convergence and Consistency Analysis for Extended Kalman Filter Based SLAM. Robotics, IEEE Transactions on, 23, 1036-1049.

Sr, C. S. 3000 manual v1. 02, June 2006. Available to Swiss-Ranger customers: http://www. swissranger. ch/customer.

Stacey, A., Jancic, M., \& Grundy, I. (2003). Particle swarm optimization with mutation, Evolutionary Computation, 2003. CEC '03. The 2003 Congress on, pp. 1425-1430.

Zwe-Lee, G. (2003). Discrete particle swarm optimization algorithm for unit commitment, Power Engineering Society General Meeting, 2003, IEEE, pp. 424. 


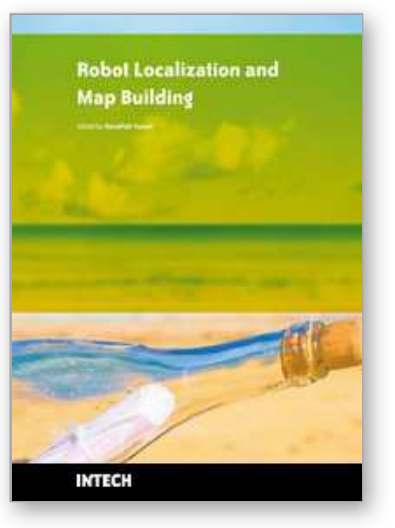

\author{
Robot Localization and Map Building \\ Edited by Hanafiah Yussof
}

ISBN 978-953-7619-83-1

Hard cover, 578 pages

Publisher InTech

Published online 01, March, 2010

Published in print edition March, 2010

Localization and mapping are the essence of successful navigation in mobile platform technology. Localization is a fundamental task in order to achieve high levels of autonomy in robot navigation and robustness in vehicle positioning. Robot localization and mapping is commonly related to cartography, combining science, technique and computation to build a trajectory map that reality can be modelled in ways that communicate spatial information effectively. This book describes comprehensive introduction, theories and applications related to localization, positioning and map building in mobile robot and autonomous vehicle platforms. It is organized in twenty seven chapters. Each chapter is rich with different degrees of details and approaches, supported by unique and actual resources that make it possible for readers to explore and learn the up to date knowledge in robot navigation technology. Understanding the theory and principles described in this book requires a multidisciplinary background of robotics, nonlinear system, sensor network, network engineering, computer science, physics, etc.

\title{
How to reference
}

In order to correctly reference this scholarly work, feel free to copy and paste the following:

Ren C. Luo and Chun C. Lai (2010). Consistent Map Building Based on Sensor Fusion for Indoor Service Robot, Robot Localization and Map Building, Hanafiah Yussof (Ed.), ISBN: 978-953-7619-83-1, InTech, Available from: http://www.intechopen.com/books/robot-localization-and-map-building/consistent-map-buildingbased-on-sensor-fusion-for-indoor-service-robot

\section{INTECH}

open science | open minds

\section{InTech Europe}

University Campus STeP Ri

Slavka Krautzeka 83/A

51000 Rijeka, Croatia

Phone: +385 (51) 770447

Fax: +385 (51) 686166

www.intechopen.com

\section{InTech China}

Unit 405, Office Block, Hotel Equatorial Shanghai

No.65, Yan An Road (West), Shanghai, 200040, China

中国上海市延安西路65号上海国际贵都大饭店办公楼 405 单元

Phone: +86-21-62489820

Fax: $+86-21-62489821$ 
(C) 2010 The Author(s). Licensee IntechOpen. This chapter is distributed under the terms of the Creative Commons Attribution-NonCommercialShareAlike-3.0 License, which permits use, distribution and reproduction for non-commercial purposes, provided the original is properly cited and derivative works building on this content are distributed under the same license. 